\title{
Evolution at 150: time for truly biological psychiatry
}

Randolph M. Nesse

\section{Summary}

Darwin's On the Origin of Species was published 150 years ago, but evolution is just now being recognised as the missing half of a truly biological psychiatry. The general framework offered by an evolutionary perspective may be as valuable as its specific applications.

\section{Declaration of interest}

None
Randolph Nesse is a Professor of Psychiatry and a Professor of Psychology at the University of Michigan where he studies evolutionary applications in medicine.

On 24 November, 150 years ago, the first copies of On the Origin of Species hit the streets. ${ }^{1}$ Darwin's core idea, natural selection, has changed our view of life and ourselves. It is so simple that Thomas Huxley famously observed 'How stupid of me not to have thought of that!? If members of a species with a certain heritable variation have more surviving offspring than others, that trait will become more prevalent over the generations, and the species will change. This explains not only the origins of different species, but also, and more important for medicine, the origins of adaptation. Natural selection explains why the body works so well. Individuals with variations that work less well - an inability to sweat, or an inability to have a panic reaction in the face of life-threatening danger - have fewer offspring. It also explains behaviour. The genes of individuals who are reckless, uninterested in mating or oblivious to their offspring will be displaced by those of individuals whose behaviour better maximises reproduction.

Evolutionary applications in medicine are growing fast. ${ }^{2}$ Some arise from new genetic data and methods, but many are coming from increased appreciation for Mayr and Tinbergen's recognition that every trait needs two different kinds of explanation: a proximate explanation of how it works; and an evolutionary explanation of how it came to be the way it is. For instance, a proximate explanation of the adrenal gland describes its structure, chemistry, regulation and ontogeny. An evolutionary explanation describes its origins and how variations associated with differences in survival and reproduction shaped it to its present form. Physiology provides the rest of medicine with an integrated understanding of structure and evolved adaptive function. The focus of biological psychiatry has been on proximate mechanisms, with only passing attention to their evolutionary-origins effects on fitness. From this perspective, biological psychiatry is making full use of only one half of biology. The other half is available in the field of animal behaviour, where every object of study receives both evolutionary and proximate explanations. For instance, animal behaviour studies investigate the brain mechanisms that regulate foraging in tight conjunction with exquisite mathematical models of how strategy variations influence fitness in different environments.

It has become clear recently that evolution can explain not only why the body usually works well, but also why it is vulnerable to diseases. Diseases are not shaped by selection; they have no evolutionary explanation. However, aspects of the body that leave it vulnerable to disease do need an evolutionary explanation. Why didn't natural selection make the birth passage wider and mood and anxiety less prone to excess responses? Sometimes selection simply cannot do better. Usually, however, the explanation also involves trade-offs, mismatch to the modern environment, fastevolving pathogens or the costs of protective responses. The enterprise of addressing such questions is one important part of Darwinian medicine. ${ }^{3}$

Related evolutionary ideas are being applied to psychiatry, with a new textbook ${ }^{4}$ augmenting previous volumes ${ }^{5,6}$ and scores of articles. ${ }^{7}$ There is no room here for even a summary. It is possible, however, to consider the benefits of asking evolutionary questions about why we are vulnerable to mental disorders, using schizophrenia and depression to illustrate the opportunities.

\section{Schizophrenia}

The evolutionary question about schizophrenia is why selection has not eliminated the genes for this highly heritable disease that so dramatically decreases fitness. Attempts to find some fitness benefit associated with schizophrenia mainly illustrate the problem of posing evolutionary explanations without trying to test them against alternatives. Now we can look for the genes themselves. A decade ago, many thought we would soon find the responsible culprits. We not only have failed to find them, it is increasingly clear that there are no common genes with large effects on schizophrenia.

Now what? Larger samples and better biometrics will assemble small effects into larger influences, and they may point to crucial metabolic pathways. However, an evolutionary geneticist points out that there are evolutionary reasons why we should not expect to find common genes with significant negative effects: 'Geographically dispersed or common risk alleles are older and more likely to be repeatedly detected . . . But, their widespread dispersion indicates that those alleles are benign (at least in regard to fitness history), so if they are associated with disease the causal finger actually points to recent environmental change rather than primarily to genetic etiology. ${ }^{8}$ This changes the model from a search for defective genes to recognition that many genes causing highly heritable common disorders are normal variations that cause disease only when they interact with aspects of the environment that are novel in the past few hundred generations. For schizophrenia, aspects of social life that influence fitness are prime candidates, especially social selection, which can shape extreme traits that give fitness advantages to those preferred as friends and group members. ${ }^{9}$ 
However, new evidence points to a strong role in schizophrenia for variations in the locations and number of copies of a gene. This poses a different evolutionary question: how can large effects from many different loci cause similar syndromes? Darwinian medicine offers a possible approach by asking why selection has left the body vulnerable. For instance, the distal head of the radius often breaks on falling forwards, as parents of skateboarders know all too well. Why hasn't natural selection made the radius more robust? Because a thicker radius would limit wrist mobility, and the ability to throw things hard and fast. Many genes no doubt contribute to the risk of fracture. In addition to those that influence radius thickness, risk will also come from genes that influence bone structure, calcium metabolism, risk-taking, and a tendency to love alcohol or hate milk.

Does some aspect of the human cognitive apparatus leave it vulnerable to schizophrenia? I have wondered whether cliff-edge effects might be involved. ${ }^{10} \mathrm{~A}$ useful trait may increase fitness as it gets closer to an extreme, but at some point, catastrophic failure becomes more likely. For instance, selective breeding of race horses shapes long, light, thin leg bones. This increases speed, but increases the risk of a fatal fracture. Has some cognitive trait given humans such a huge advantage that it has been selected rapidly and pushed close to a fitness cliff edge? Like vulnerability to fracture, its heritability would likely arise from many small effects. I am by no means confident that this is correct, but it illustrates how asking evolutionary questions can bring up new possibilities.

\section{Depression}

The evolutionary question about depression is very different. Most people never have symptoms of schizophrenia, but nearly everyone has periods of low mood. Is low mood always abnormal, like seizures, or is it an evolved protective response, like fever, cough and anxiety? The rest of medicine makes this distinction sharply and intuitively. As a result, physicians no longer diagnose cough disorder, they instead diagnose pneumonia or congestive heart failure. There is nothing useful about severe depression, but is it a disease like type 1 diabetes, or is it, like chronic pain, a dysregulation in a useful evolved response system? It is important to find out. If the capacity for mood is useful in certain situations, then we must discover its evolutionary origins and functions before we can understand depression.

This has implications for diagnosis, research and treatment. The architects of new diagnostic manuals are seeking new ideas. They could consider bringing the evolutionary half of biology to bear on the problem. If the normality of mood depends on the situation, as it certainly does, then considering the situation is essential before making a depression diagnosis except in extreme cases. ${ }^{11}$ This would provide a scientific foundation for validity, but reliability would plummet. An evolutionary perspective may be more helpful for explaining why finding a satisfactory nosology has been so difficult, rather than offering easy solutions.

Asking evolutionary questions about mood could also assist the search for aetiology. It emphasises the importance of identifying the mechanisms that mediate and regulate normal low mood as a foundation for understanding abnormal mood. It also suggests that environmental effects are likely to be mediated by processing information far more subtle than the amount of 'stress' a person encounters. Methods to assess the motivational structures of people's lives - what a person is doing to get crucial resources, trade-offs between different enterprises, and why goals cannot be reached - are essential to test the hypothesis that depression symptoms arise when people are trapped pursuing unreachable goals. ${ }^{12}$

A long campaign has tried to convince the public that drug treatment is appropriate because depression is a brain disease. However, people deserve relief from depression whether or not it arises from a brain abnormality. Physicians in the rest of medicine use medications to relieve pain, whether it is aroused by an appropriate stimulus, or by some abnormality in the pain system. They prescribe only, however, after looking carefully for the cause. Most psychiatrists attend carefully to aspects of their patient's lives that may contribute to depression, and do what they can to deal with any causes they find. An evolutionary perspective helps to explain why this is essential.

Asking evolutionary questions about why we are vulnerable to disorders such as schizophrenia and depression does not offer quick explanations, but it does suggest new possibilities that deserve study. Darwin's principle of natural selection has been available for 150 years. Now would be a good time to start addressing evolutionary questions about all aspects of mental disorders. This will be difficult work, but it can provide us, and our patients, with a truly biological psychiatry.

Randolph M. Nesse, MD, The University of Michigan, Research Center for Group Dynamics, ISR, 530 Church Street, Ann Arbor, MI 48109-1043, USA Email: nesse@umich.edu

First received 17 Sep 2009, accepted 19 Oct 2009

\section{Acknowledgements}

Thanks to members of the University of Michigan Research Track Seminar for useful suggestions

\section{References}

1 Darwin C. On the Origin of Species. John Murray, 1859

2 Nesse RM, Stearns SC. The great opportunity: evolutionary applications to medicine and public health. Evolutionary Applications 2008; 1: 28-48.

3 Nesse RM, Williams GC. Why We Get Sick: The New Science of Darwinian Medicine. Times Books, 1994.

4 Brüne M. Textbook of Evolutionary Psychiatry: The Origins of Psychopathology. Oxford University Press, 2008.

5 McGuire MT, Troisi A. Darwinian Psychiatry. Harvard University Press, 1998.

6 Wenegrat B. Sociobiological Psychiatry: Normal Behavior and Psychopathology. Lexington Books, 1990

7 Nesse RM. Evolutionary psychology and mental health. In The Evolutionary Psychology Handbook (ed D Buss): 903-27. John Wiley \& Sons, 2005.

8 Weiss KM. Tilting at Quixotic Trait Loci (QTL): an evolutionary perspective on genetic causation. Genetics 2008; 179: 1741-56.

9 Nesse RM. Runaway selection for displays of partner value and altruism. Biol Theory 2007; 2: 143-55.

10 Nesse R. Cliff-edged fitness functions and the persistence of schizophrenia (commentary). Behavioral and Brain Sciences 2004; 27: 862-3.

11 Horwitz AV, Wakefield JC. The Loss of Sadness: How Psychiatry Transformed Normal Sorrow into Depressive Disorder. Oxford University Press, 2007.

12 Nesse RM. Explaining depression: neuroscience is not enough, evolution is essential. In Understanding Depression. A Translational Approach (eds CM Pariante, RM Nesse, DJ Nutt, et al): 17-36. Oxford University Press, 2009 . 